\title{
CT imaging features of different clinical types of COVID-19 calculated by Al system: a Chinese multicenter study
}

\author{
Xiaofei Hu${ }^{1 \#}$, Wenbing Zeng ${ }^{2,3 \#}$, Yuhan Zhang ${ }^{1 \#}$, Zhiming Zhen ${ }^{1}$, Yalan Zheng ${ }^{1}$, Lin Cheng', \\ Xianqi Wang ${ }^{1}$, Haoran Luo ${ }^{1}$, Shu Zhang ${ }^{4}$, Zifeng $\mathrm{Wu}^{4}$, Zeyu Sun ${ }^{4}$, Xiuli $\mathrm{Li}^{4}$, Yang $\mathrm{Cao}^{5}, \mathrm{Ming} \mathrm{Xu}^{6}$, \\ Jian Wang ${ }^{1}$, Wei Chen ${ }^{1}$
}

${ }^{1}$ Department of Radiology, First Affiliated Hospital of the Army Medical University (Southwest Hospital), Chongqing, China; ${ }^{2}$ Department of Radiology, Chongqing University Three Gorges Hospital, Wanzhou, China; ${ }^{3}$ Department of Radiology, Chongqing Three Gorges Central Hospital, Chongqing Three Gorges Central Hospital, Wanzhou, China; ${ }^{4}$ Deepwise AI Lab, No.8 Haidian avenue, Sinosteel International Plaza, Beijing, China; ${ }^{5}$ Department of Radiology, Dianjiang People's Hospital of Chongqing, Dianjiang, China; ${ }^{6}$ Department of Radiology, Chongqing Tongnan County Peoples Hospital, Tongnan, China

Contributions: (I) Conception and design: J Wang, W Chen; (II) Administrative support: None; (III) Provision of study materials or patients: X Hu; (IV) Collection and assembly of data: W Zeng, Y Cao, M Xu; (V) Data analysis and interpretation: S Zhang, Z Sun, X Li; (VI) Manuscript writing: All authors; (VII) Final approval of manuscript: All authors.

\#These authors contributed equally to this work.

Correspondence to: Jian Wang, MD; Wei Chen, MD. Department of Radiology, First Affiliated Hospital of the Army Medical University (Southwest Hospital), Gaotanyan Street, Chongqing 400038, China. Email: wangjian811@gmail.com; cwjxl_2006@163.com.

Background: The study is designed to explore the chest CT features of different clinical types of coronavirus disease 2019 (COVID-19) pneumonia based on a Chinese multicenter dataset using an artificial intelligence (AI) system.

Methods: A total of 164 patients confirmed COVID-19 were retrospectively enrolled from 6 hospitals. All patients were divided into the mild type (136 cases) and the severe type (28 cases) according to their clinical manifestations. The total CT severity score and quantitative CT features were calculated by AI pneumonia detection and evaluation system with correction by radiologists. The clinical and CT imaging features of different types were analyzed.

Results: It was observed that patients in the severe type group were older than the mild type group. Round lesions, Fan-shaped lesions, crazy-paving pattern, fibrosis, "white lung", pleural thickening, pleural indentation, mediastinal lymphadenectasis were more common in the CT images of severe patients than in the mild ones. A higher total lung severity score and scores of each lobe were observed in the severe group, with higher scores in bilateral lower lobes of both groups. Further analysis showed that the volume and number of pneumonia lesions and consolidation lesions in overall lung were higher in the severe group, and showed a wider distribution in the lower lobes of bilateral lung in both groups.

Conclusions: Chest CT of patients with severe COVID-19 pneumonia showed more consolidative and progressive lesions. With the assistance of AI, CT could evaluate the clinical severity of COVID-19 pneumonia more precisely and help the early diagnosis and surveillance of the patients.

Keywords: Coronavirus infections; pneumonia, viral; severity of illness index; tomography, X-ray computed; artificial intelligence (AI)

Submitted Apr 05, 2020. Accepted for publication Aug 21, 2020.

doi: $10.21037 /$ jtd-20-1584

View this article at: http://dx.doi.org/10.21037/jtd-20-1584 


\section{Introduction}

The ongoing coronavirus disease 2019 (COVID-19) have had a profound global and individual burden (1). Since the detection of the coronavirus in late December 2019, the COVID-19 continued to erupt in over 213 countries, areas, and territories at the time of writing.

Similar to other coronaviral pneumonia such as severe acute respiratory syndrome caused by Severe Acute Respiratory Syndrome Coronavirus (SARS-CoV) and the Middle East Respiratory Syndrome Coronavirus (MERSCoV), COVID-19 can also cause fever, cough, and shortness of breath at the time of onset and lead to acute respiratory distress syndrome (ARDS) (2). On admission, most cases of COVID-19 were classified as mild (81\%). However, 14\% were severe, and $5 \%$ were critical (3). For mild patients, general isolation and symptomatic treatment were available, and ICU-care was needed if the condition worsens rapidly, such to reduce the mortality and alleviate the shortage of medical resources. And severely-ill patients progressed rapidly to acute respiratory failure, acute respiratory distress syndrome, metabolic acidosis, coagulopathy, and septic shock (2). The overall case-fatality rate (CFR) was estimated to be $2.3-3.06 \%$, but the CFR was up to $49.0 \%$ among critical cases (3). And severe pneumonia was independently associated with either the admission to intensive care unit, mechanical ventilation, or death (2). Therefore, early identification of risk factors for severe illness facilitated appropriate supportive care and prompt access to ICU if necessary, and the data on the clinical characteristics and CT findings of severely-ill patients with SARS-CoV-2 infection are scarce, but are of paramount importance to reduce mortality.

Previous studies have only described the general CT findings of patients of COVID-19 (4-6). In this article, we summarized qualitative and quantitative CT features of patients with different severity of COVID-19 based on a Chinese multicenter dataset, aiming to provide clinical value of early diagnosis and evaluation of its severity. It is worth mentioning that the quantitative CT features were performed by an artificial intelligence (AI) pneumonia detection and evaluation system and then adjusted by multiple junior radiologists, which made them relatively more accurate than gross visual evaluation.

We present the following article in accordance with the STROBE reporting checklist (available at http://dx.doi. org/10.21037/jtd-20-1584).

\section{Methods}

This study was approved by the Ethics of Committees of Southwest Hospital (No. KY2020036). Informed consent for this retrospective study was waived. The anonymous data was collected and analyzed. The study was conducted in accordance with the Declaration of Helsinki (as revised in 2013) (7).

\section{Patients and chest CT}

From January 28, 2020, until February 24, 2020, adult patients admitted to six hospitals of Chongqing in China with confirmed COVID-19 and who underwent chest CT were enrolled in our study. Real-time fluorescence polymerase chain reaction of nasopharyngeal swab or sputum samples methods were positive for the COVID-19 twice. In addition to age and sex, clinical information collected included severity.

All scans were performed without intravenous contrast with the patient in the supine position during endinspiration. And at least one chest CT was performed at the onset of the disease. Only the initial chest CTs were evaluated; if a patient had a follow-up CT during the study time window, it was not analyzed for this study. All the patients were divided into two types according to the clinical symptom (8). Mild type: Patients with symptoms of fever, respiratory symptom, etc. and imaging findings of pneumonia. Severe type: Patients who meet any of the following criteria: (I) respiratory distress, respiratory rate $\geq 30 / \mathrm{min}$; (II) oxygen saturation at rest $\leq 93 \%$; (III) $\mathrm{PaO}_{2} / \mathrm{FiO}_{2} \leq 300 \mathrm{mmHg}(1 \mathrm{mmHg}=0.133 \mathrm{kPa})$. Patients who meet any of the following criteria were excluded: (I) respiratory failure and mechanical ventilation are required; (II) shock; (III) multiple organ failure except lung, and intensive care unit is required.

\section{CT review and analysis}

All CT images were reviewed by two radiologists who had approximately 4 and 5 years of experience in thoracic radiology, respectively. Imaging was reviewed independently and final decisions reached by consensus. For disagreement 
between the two primary radiologist interpretations, a third fellowship-trained cardiothoracic radiologist with 25 years of experience adjudicated a final decision. No negative control cases were examined and no blinding occurred.

The major qualitative CT demonstrations were described using internationally standard nomenclature defined by the Fleischner Society glossary and peer-reviewed literature on viral pneumonia, using terms including ground glass opacity (GGO), crazy-paving pattern, and consolidation $(9,10)$.

A semi-automatic AI pneumonia detection and evaluation system was used to extract CT features and quantitatively estimate the pulmonary involvement of these abnormalities. The system used in our experiment to detect and segment lung abnormalities was built according to the pre-trained deep learning models in our previous study (10-12). The detection and evaluation process of this system was divided into five steps. (I) Lesion detection, an MVP-Net (MultiView FPN with Position-aware attention) which was trained on the NIH DeepLesion dataset and achieved stateof-the-art performance was used to realize the detection of lung abnormalities (11). The result of detection mainly included two patterns: consolidation and ground glass opacity. (II) Automatic lesion segmentation, a 2D U-Net model trained on a local dataset of about 8,000 lung CT images was used to segment detected lesions (12). (III) Manual correction, the automatic segmentation results of lesions were firstly adjusted by multiple junior radiologists, then a senior radiologist reviewing the segmentation results of AI system and junior radiologists to provide the final contours of inflammatory lesions (Figure 1). (IV) Lesion

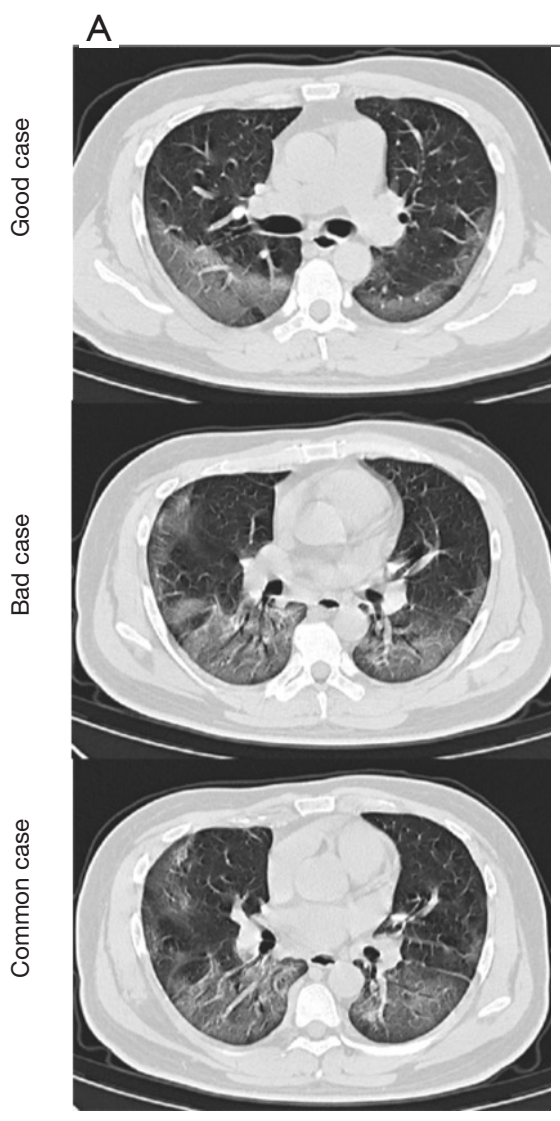

(a) Origin
B

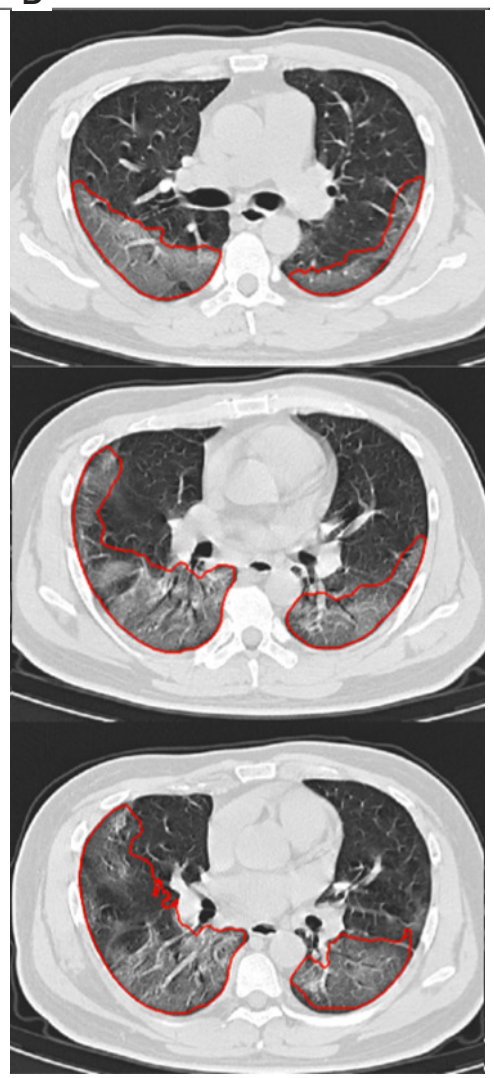

(b) Automatic

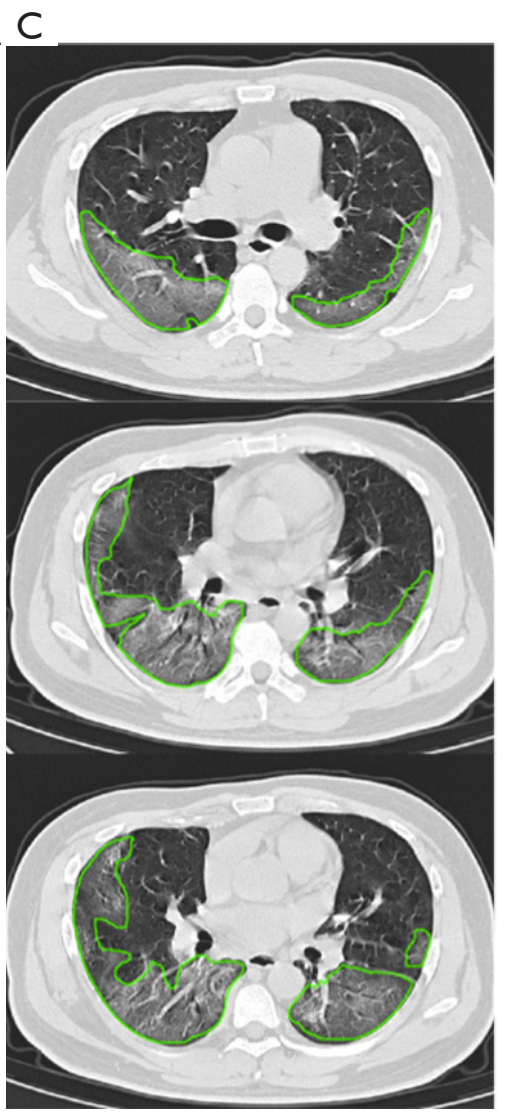

(c) Manual

Figure 1 The comparison of lesion contours from automatic segmentation and manual correction. (A) The original CT image; (B) automatic segmentation results of lesions by AI pneumonia detection system (red outline); (C) the results of manual correction by radiologists (green outline). Besides, some examples of different performances are selected for comparison, from top to bottom are good case, common case and bad case respectively. 
Table 1 Clinical manifestations of COVID-19 patients with different clinical type

\begin{tabular}{|c|c|c|c|}
\hline Variable & Mild type $(n=136)$ & Severe type $(n=28)$ & $\mathrm{P}$ \\
\hline Gender, n (\%) & & & $0.084^{b}$ \\
\hline Male & $70(51.5)$ & $20(71.5)$ & \\
\hline Female & $66(48.5)$ & $8(28.5)$ & \\
\hline Cough, n (\%) & $92(67.64)$ & $21(75.0)$ & $0.58^{\mathrm{b}}$ \\
\hline White blood cell count $\left(\times 10^{9} / \mathrm{L}\right)$ & 5.1 (4.0 to 6.5$)$ & $5.6(4.2$ to 7.7$)$ & $0.273^{\mathrm{a}}$ \\
\hline Neutrophil count $\left(\times 10^{9} / \mathrm{L}\right)$ & $3.4(2.5$ to 4.8$)$ & 4.5 (2.9 to 6.5$)$ & $0.066^{\mathrm{a}}$ \\
\hline Lymphocyte count $\left(\times 10^{9} / L\right)$ & $1.58 \pm 4.31$ & $1.14 \pm 0.49$ & $0.596^{c}$ \\
\hline
\end{tabular}

${ }^{a}$, Mann Whitney $U$ test; ${ }^{b}$, chi-square test; ${ }^{c}$, $t$-test. Data are presented as $\mathrm{n}(\%)$, mean \pm SD or median (25th to 75 th percentile). COVID-19, coronavirus disease 2019.

localization, pulmonary lobes were segmented by a pretrained lobe segmentation model and intersected with the final segmentation contours of lung abnormalities to achieve the localization of lesions in each lobe (13). (V) Pneumonia assessment, the lesion and lobe information were extracted to realize quantitative analysis of pneumonia. Furthermore, a lung severity score was used to quantitatively estimate the pulmonary involvement of all these abnormalities based on the area involved (4). Each of the 5 lung lobes was scored, from 0 to 4 as: 0 , no involvement; $1,1-25 \%$ involvement; 2, 26-50\% involvement; 3, 51-75\% involvement; 4, $76-100 \%$ involvement. The overall lung "total severity score" was the sum of the 5 lobe scores and ranged from 0 (no involvement) to 20 (maximum involvement). In this experiment, we also calculated the distribution of these quantitative analysis results and lung severity score of patients with different clinical types.

\section{Statistical methods}

Statistical analyses were performed using IBM SPSS Statistics Software (version 24; IBM, New York, USA). Quantitative data were presented as mean \pm standard deviation (minimum-maximum) and the counting data were presented as the percentage of the total unless otherwise specified. Quantitative data were compared using the $t$ test or Mann Whitney $U$ test, according to the normal distribution assessed by the Shapiro-Wilk test. The comparisons of the counting data of clinical and CT features were evaluated using chi-square test or Fisher's exact test. A P value of $<0.05$ was defined as statistical significance.

\section{Results}

\section{Clinical manifestations of COVID-19 patients with different clinical types}

This study included 164 patients diagnosed as COVID-19, of which 136 were with mild type $(82.9 \%)$ and 28 were with severe type (17.1\%). Patients in the severe type group were with higher age than patients in the mild type (median age, 58 vs. $46, \mathrm{P}<0.001)$. There was a higher proportion of males in both groups $(51.5 \%, 71.5 \%)$, and most of the patients in this series were with fever $(63.9 \%, 78.5 \%)$ and cough $(67.64 \%, 75.0 \%)$ as the main clinical manifestations. There was a marked and significant increase in the C-Reactive Protein $(C R P)$ in the severe type group $(\mathrm{P}<0.001)$. However, there were no significant differences in white blood cell count, neutrophils count and lymphocyte count between the two groups (Table 1).

\section{Distribution of lung abnormalities in COVID-19 patients with different clinical types}

Overall, common features of this series were found on the chest CT images of all the patients: 139 (84.7\%) were with bilateral disease, 147 (89.6\%) with multiple lesions, $156(95.1 \%)$ with lesions located in the subpleural area, $117(71.3 \%)$ with ground-glass opacities and consolidative 
Table 2 Distribution of lung abnormalities in COVID-19 patients with different clinical types

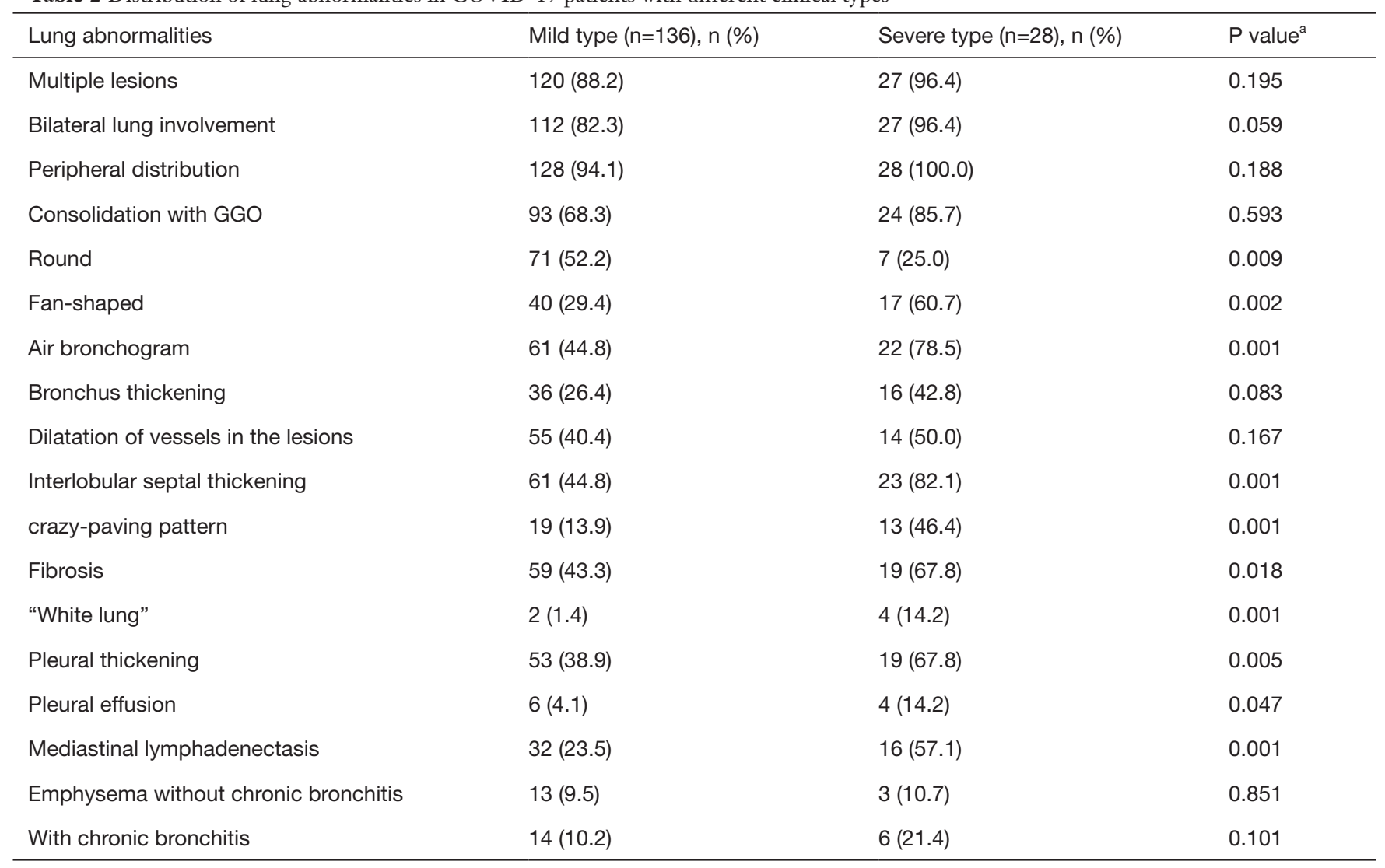

a, chi-square test. COVID-19, coronavirus disease 2019; GGO, ground glass opacities.

pulmonary opacities. Meanwhile, bronchial wall thickening $(52,31.7 \%)$, fibrosis $(78,47.5 \%)$ and the vascular enlargement, $(69,42.1 \%)$ were found in nearly half of the patients. The crazy-paving pattern $(42,25.6 \%)$ and "white lung" $(6,3.6 \%)$ were rare, and mostly had no emphysema without chronic bronchitis $(148,90.2 \%)$, chronic bronchitis $(144,87.8 \%)$, pleural traction $(137,83.5 \%)$ and mediastinal lymph node enlargement (116, 70.7\%).

The distribution of lung abnormalities between different clinical types was detailed in Table 2. Significant differences were observed between patients with mild type and severe type in some qualitative CT features. Fan-shaped lesions (17, 60.7\%), air bronchogram (22, 78.5\%), interlobular septal thickening $(23,82.1 \%)$, crazy-paving pattern (13, $46.4 \%)$, fibrosis $(19,67.8 \%)$, "white lung" (4, 14.2\%), pleural effusion (4, 14.2\%), pleural thickening (19, 67.8\%), mediastinal lymphadenectasis $(16,57.1 \%)$ were more common in the patients with severe type than in mild type (Figures 2,3).

\section{The severity scores of COVID-19 patients with different clinical types}

The severity scores of different clinical types were detailed in Table 3. Mann-Whitney $U$ test showed significant difference between the mild type versus the severe type for the total lung severity score $(\mathrm{P}<0.0001$, Figure 4$)$, and $t$-test showed significant difference in severity score in each lobe $(\mathrm{P}=0.0003,<0.0001,0.0002,<0.0001$ and 0.0001 , respectively). Besides, for both the two types, the severity score was significantly different between the left upper lobe versus the left lower lobe, the right upper lobe versus the right lower lobe, and the right middle lobe versus the right lower lobe $(\mathrm{P}<0.0001$ for the mild type; $0.0007,0.0048$ and 

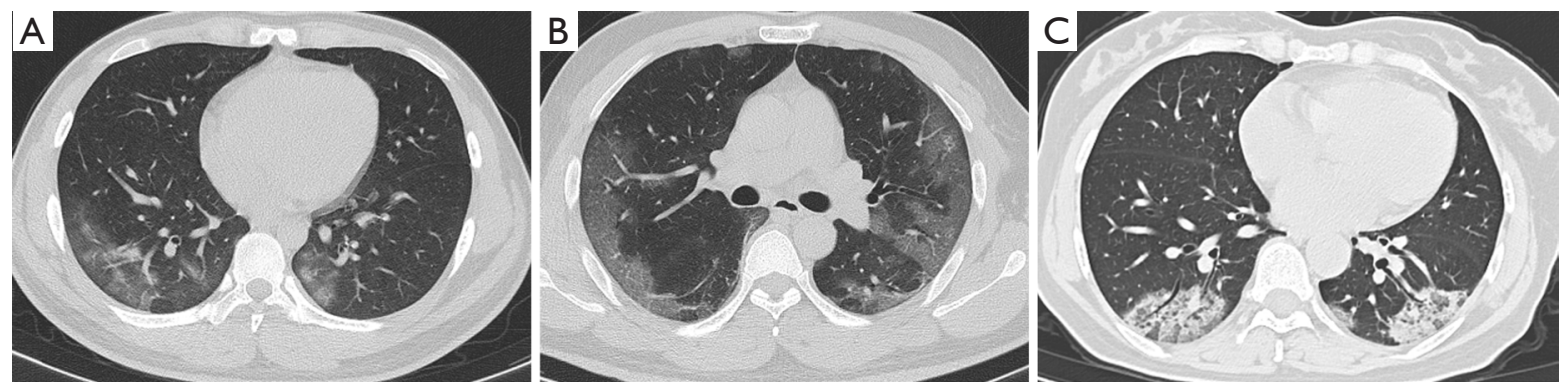

Figure 2 Examples of chest CT findings in patients with mild coronavirus disease 2019 pneumonia. (A) Male, 20 years old with fever and cough for 3 days. Axial chest CT shows subpleural consolidative opacities with a ring of ground glass opacities (GGO) in the lower lobe of bilateral lung. (B) Male, 30 years old, with fever and cough for 4 days. Axial chest CT shows bilateral areas of peripheral GGO with minimal consolidation and interlobular septal thickening. (C) Female, 56 years old, presenting with fever and cough for 1 week. Axial chest CT shows a "crazy-paving" pattern as manifested by bilateral lower lobe consolidative opacification with some ground-glass opacities, interlobular septal thickening and intralobular lines.
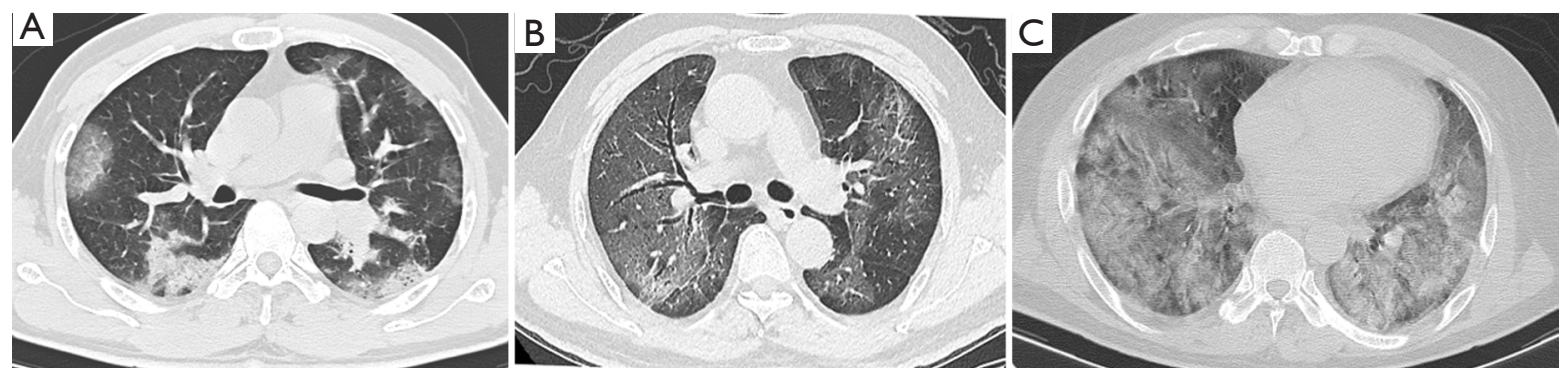

Figure 3 Examples of chest CT findings in patients with severe coronavirus disease 2019 pneumonia. (A) Male, 53 years old with fever and cough for 5 days. Axial chest CT shows bilateral subpleural opacities including GGO, consolidative opacities with a ring of GGO and consolidative opacification. (B) Male, 52 years old, with fever and cough for 6 days. Axial chest CT shows extensive ground-glass opacities in both lungs, involving almost the entire right lung, and most of the left lung, with air bronchograms. (C) Male, 56 years old, presenting with fever and cough for 1 week. Axial chest CT shows extensive consolidative opacities in both lungs, involving almost the entire bilateral lung, giving a white lung appearance.

0.0249 for the severe type respectively).

\section{Quantitative CT features of main patterns in different clinical types}

The details of quantitative CT features of main patterns were shown in Table 4. For the comparison between the two clinical types, some features showed significant difference: volume of pneumonia lesions $(\mathrm{P}<0.0001)$, volume of consolidation $(\mathrm{P}<0.0001)$, number of consolidation ( $\mathrm{P}=0.0155)$, volume of $\mathrm{GGO}(\mathrm{P}=0.0304)$, number of lobes affected $(\mathrm{P}=0.0133)$, volume ratio of pneumonia lesions for each lobe (all $\mathrm{P}<0.0001)$ the total ratio $(\mathrm{P}<0.0001)$, volume ratio of consolidation for each lobe $(\mathrm{P}=0.0002,<0.0001$,
$<0.0001,0.0002,<0.0001)$ the total ratio $(\mathrm{P}<0.0001)$ and the total volume ratio of $\mathrm{GGO}(\mathrm{P}=0.0298)$.

Besides, there were some features showing significant difference when compared among different locations: volume ratio of pneumonia lesions between the left upper lobe versus the left lower lobe for both the two types $(\mathrm{P}<0.0001$ both $)$, the right upper lobe versus the right lower lobe $(\mathrm{P}<0.0001$ and $\mathrm{P}=0.0027)$ and the right middle lobe versus the right lower lobe $(\mathrm{P}<0.0001$ and $\mathrm{P}=0.0169)$; volume ratio of consolidation between the left upper lobe versus the left lower lobe for both the two types $(\mathrm{P}<0.0001$ and $\mathrm{P}=0.0006$ ), the right upper lobe versus the right lower lobe $(\mathrm{P}<0.0001$ and $\mathrm{P}=0.0027)$ and the right middle lobe versus the right lower lobe $(\mathrm{P}<0.0001$ and $\mathrm{P}=0.0021)$; 
Table 3 The severity scores of COVID-19 patients with different clinical types

\begin{tabular}{llll}
\hline Severity score & Mild type $(n=136)$ & Severe type $(n=28)$ & $P$ \\
\hline Total lung severity score & $4.65 \pm 2.48$ & $9.43 \pm 4.43$ & $<0.0001$ \\
Severity score in each lobe & & & 0.0003 \\
Left upper lobe & $0.85 \pm 0.58^{\mathrm{a}}$ & $1.64 \pm 0.99^{\mathrm{a}}$ & $<0.0001$ \\
Left lower lobe & $1.16 \pm 0.84$ & $2.18 \pm 0.90$ & 0.0002 \\
Right upper lobe & $0.74 \pm 0.57^{\mathrm{b}}$ & $1.64 \pm 1.10^{\mathrm{b}}$ & $<0.0001$ \\
Right middle lobe & $0.71 \pm 0.63^{\mathrm{c}}$ & $1.79 \pm 1.17^{\mathrm{c}}$ & 0.0001 \\
Right lower lobe & $1.19 \pm 0.76$ & $2.18 \pm 1.16$ & 0 \\
\hline
\end{tabular}

${ }^{a}, t$-test showed significant difference between the left lower lobe and the left upper lobe $(\mathrm{P}<0.05) ;{ }^{\mathrm{b}}, t$-test showed significant difference between the right upper lobe and the right lower lobe $(\mathrm{P}<0.05) ;{ }^{c}, t$-test showed significant difference between the right middle lobe and the right lower lob $(\mathrm{P}<0.05)$. Quantitative data were presented as mean \pm standard deviation. COVID-19, coronavirus disease 2019.

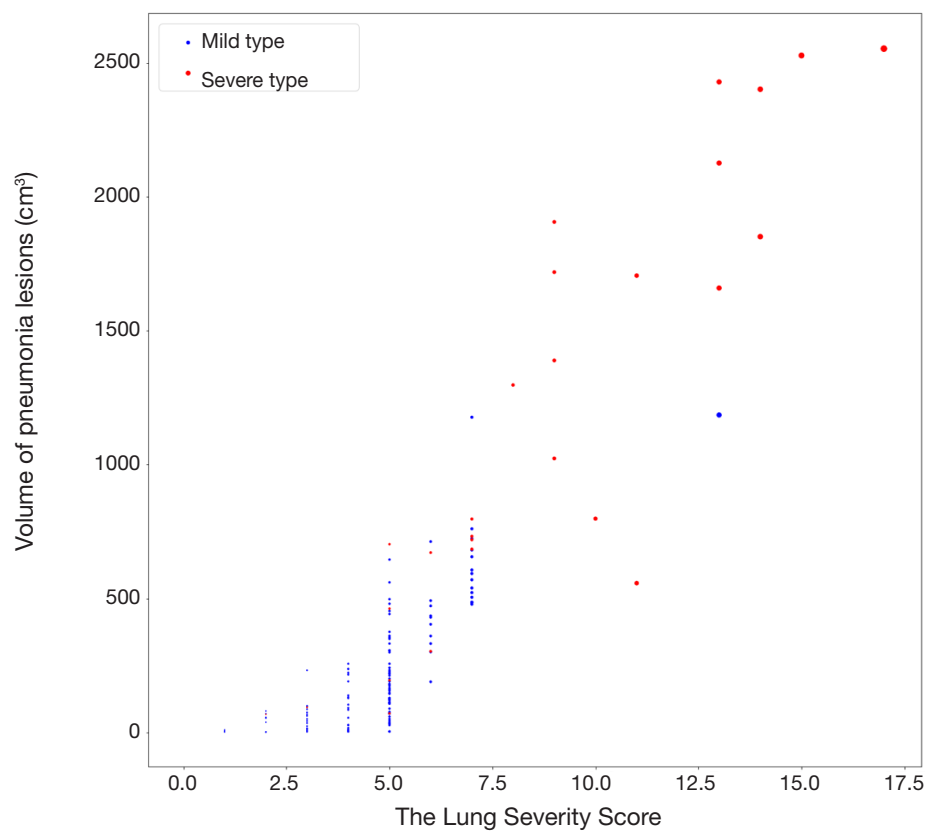

Figure 4 The scatter plot for the relationship between volume of pneumonia lesions and the lung severity score. Horizontal axis is the lung severity score for assessing the degree of pneumonia and vertical axis is the volume of pneumonia lesions. In the scatter plot, the blue dots represent patients with mild type and the red dots represent patients with severe type.

volume ratio of GGO between left upper lobe versus the left lower lobe for the mild type $(\mathrm{P}<0.0001)$, the right upper lobe versus the right lower lobe for the mild type $(\mathrm{P}<0.0001)$, and the right upper lobe versus the right middle lobe for both the two types $(\mathrm{P}=0.0190$ and $\mathrm{P}=0.0405)$.

\section{Discussion}

In this study, 164 patients were diagnosed as COVID-19 with 136 of mild type (82.9\%) and 28 of severe type (17.1\%), the proportions of which were consistent with the previous study $(81 \%$ of confirmed cases were mild while $14 \%$ were severe) (3). However, patients in the severe type group were older than in the mild type group(median age, $58 \mathrm{vs}$. 46 years), and previous study has also found similar age distribution (severe pneumonia $v s$. mild pneumonia: $58 v s$. 45) (2). It may be attributed to the low immune function and is vulnerable to the virulence of the virus in patients of 
Table 4 Quantitative CT features of main patterns in different clinical types

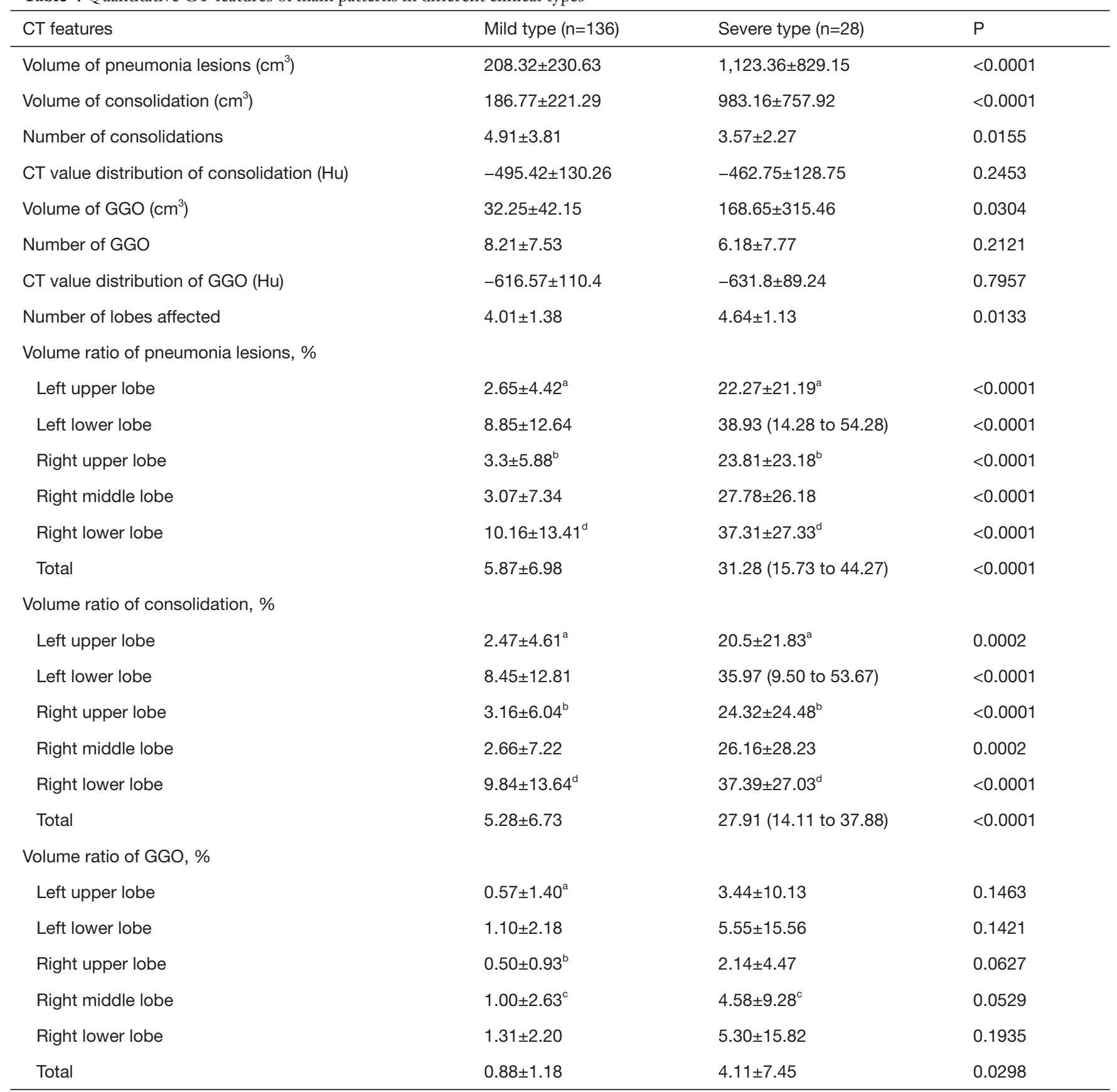

Quantitative data were presented as mean \pm standard deviation or median (25th to 75th percentile). Numbers in parentheses were percentage of the total. ${ }^{a}$, Wilcoxon test showed significant difference between the left lower lobe and the left upper lobe $(\mathrm{P}<0.05)$; ${ }^{\mathrm{b}}$, $t$-test showed significant difference between the right upper lobe and the right lower lobe $(\mathrm{P}<0.05) ;{ }^{c}, t$-test showed significant difference between the right upper lobe and the right middle lobe $(\mathrm{P}<0.05) ;{ }^{d}, t$-test showed significant difference between the right middle lobe and the right lower lobe $(\mathrm{P}<0.05)$. GGO, ground glass opacities; Hu, Hounsfield unit. 
old ages. What's more, patients in the severe type group had elevated C-reactive protein levels compared with mild cases, which was also consistent with the previous study (2).

Chest CT is a vital component in not only the diagnostic procedure but also the severity evaluation for COVID-19 patients. CT findings of this study largely concur with early radiology investigative efforts insofar as this pattern of groundglass and consolidative pulmonary opacities, often with a bilateral and peripheral lung distribution, is emerging as the chest CT hallmark of COVID-19 infection (6,9,14-16). However, obvious differences in qualitative CT features between the two types were observed. The higher incidence of the initial acute opacities such as ground-glass opacities with peripheral lung distribution occurred in patients with mild type, the more progressive, organizing and fibrosis changes such as consolidation, "crazy" paving pattern and "white lung" were found in patients with severe type. Moreover, the notable higher incidence of ancillary chest CT findings such as lymphadenectasis, pleural effusions, pleural thickening occurred in patients with the severe type.

The apparent trend indicated that an initial (often infectious or inflammatory) acute insult causes ground-glass opacities that may coalesce into dense consolidative lesions in mild stage, and then progressively evolve in often a more linear fashion even severe lung damage such as "white lung" in severe stage (17). The pathological study also showed differences in pathological change of mild and severe patients. Mild lung damage exhibited edema, the exudation of alveoli and focal hyperplasia of pneumocytes, without prominent hyaline membranes (18). While severe lung damage showed that interest mononuclear inflammatory infiltrates were seen in both lungs, and multihull syncytial cells were identified in the intra-alveolar spaces, showing viral cytopathic-like changes (19). And pulmonary oedema with hyaline membrane formation suggestive of early-phase ARDS.

Indeed, to better guide treatment and prognostic strategies, the National Health Commission of the People's Republic of China has encouraged severity evaluation based on the clinical and chest radiographic progression: COVID-19 patients with radiographic progression $>50 \%$ within 48 hours should be taken as severely ill patients (8). And this posed new challenges for radiographic evaluation, especially quantitative and semi-quantitative assessment, such as lung severity score which was mentioned among numerous studies. In the current study, we provided a relatively accurate method than gross visual evaluation for the quantitative assessment, performed by an $\mathrm{AI}$ pneumonia detection and evaluation system then adjusted by multiple junior radiologists. And obvious differences in quantitative CT features between the two types were observed. The severity scores were higher in severe cases, with higher scores in bilateral lower lungs. Further analysis found that with the fusion of the lesions, the number of pneumonia lesions in overall lung was less in the severe group, but the volume of pneumonia lesions was higher, the same trend was observed in all lobes but with no difference in the number of GGO. Specific to each lobe, more lesions (including pneumonia lesions and consolidation lesions) were found in the lower lobes of bilateral lung than upper lobe (and right middle lobe) in both groups, while more GGOs were found in the middle lobe than in the upper lobe of the right lung. This analysis further confirmed severity scores result. The concentration of lesions in the lower lobes were also observed in other studies $(20,21)$, and the autopsy of patients of COVID-19 is expected to provide a good explanation.

\section{Conclusions}

In summary, this work represented that common chest CT findings in COVID-19 based on a Chinese multicenter dataset, included multiple GGOs, consolidations in both lungs, with mostly subpleural distribution. There were significant differences of both qualitative and quantitative CT features between mild and severe patients, and more consolidative and progressive lesions were found in severe patients, especially in the lower lung. And with the assistance of AI pneumonia detection and evaluation system, CT can better play its important role in the diagnosis and evaluation of COVID-19. Nevertheless, this study was a cross-sectional study and lacked longer follow up. However, we try to draw scenes of different CT characters in different disease stages. Future study is warranted to analyze the dynamic CT changes to find the radiological feature which could predict patient progression and potential complication development.

\section{Acknowledgments}

We thank all our colleagues who helped us during the current study. We greatly appreciate the kind assistance of Chongqing Wuxi County People's Hospital, Chongqing Wulong County People's Hospital, and Chongqing Yunyang County People's Hospital for their assistance in recruiting patients for our studies. We are also grateful to 
the many frontline medical staff for their dedication in the face of this outbreak, despite the potential threat to their own lives and the lives of their families.

Funding: None.

\section{Footnote}

Reporting Checklist: The authors have completed the STORBE reporting checklist. Available at http://dx.doi. org/10.21037/jtd-20-1584

Data Sharing Statement: Available at http://dx.doi. org/10.21037/jtd-20-1584

Conflicts of Interest: All authors have completed the ICMJE uniform disclosure form (available at http://dx.doi. org/10.21037/jtd-20-1584). The authors have no conflicts of interest to declare.

Ethical Statement: The authors are accountable for all aspects of the work in ensuring that questions related to the accuracy or integrity of any part of the work are appropriately investigated and resolved. This study was approved by the Ethics of Committees of Southwest Hospital (No. KY2020036). Informed consent for this retrospective study was waived. The study was conducted in accordance with the Declaration of Helsinki (as revised in 2013).

Open Access Statement: This is an Open Access article distributed in accordance with the Creative Commons Attribution-NonCommercial-NoDerivs 4.0 International License (CC BY-NC-ND 4.0), which permits the noncommercial replication and distribution of the article with the strict proviso that no changes or edits are made and the original work is properly cited (including links to both the formal publication through the relevant DOI and the license). See: https://creativecommons.org/licenses/by-nc-nd/4.0/.

\section{References}

1. Zhu N, Zhang D, Wang W, et al. A Novel Coronavirus from Patients with Pneumonia in China, 2019. N Engl J Med 2020;382:727-33.

2. Guan WJ, Ni ZY, Hu Y, et al. Clinical Characteristics of Coronavirus Disease 2019 in China. N Engl J Med 2020;382:1708-20.

3. Wu Z, McGoogan JM. Characteristics of and Important
Lessons From the Coronavirus Disease 2019 (COVID-19)

Outbreak in China: Summary of a Report of 72314

Cases From the Chinese Center for Disease Control and Prevention. JAMA 2020;323:1239-42.

4. Chung M, Bernheim A, Mei X, et al. CT Imaging Features of 2019 Novel Coronavirus (2019-nCoV). Radiology 2020;295:202-7.

5. Ng MY, Lee EY, Yang J, et al. Imaging profile of the COVID-19 infection: radiologic findings and literature review. Radiol Cardiothorac Imaging 2020;2:e200034.

6. Pan Y, Guan H, Zhou S, et al. Initial CT findings and temporal changes in patients with the novel coronavirus pneumonia (2019-nCoV): a study of 63 patients in Wuhan, China. Eur Radiol 2020;30:3306-9.

7. Aresté N, Salgueira M. World Medical Association Declaration of Helsinki: ethical principles for medical research involving human subjects. JAMA 2013;310:2191-4.

8. General Office of National Health Committee CNHC. Diagnosis and treatment of pneumonitis caused by new coronavirus (trial version 6) (2020-02-18) Available online: http://www.nhc.gov.cn/xcs/zhengcwj/202002/8334a8326d d94d329df351d7da8aefc2.shtml

9. Hansell DM, Bankier AA, MacMahon H, et al. Fleischner Society: glossary of terms for thoracic imaging. Radiology 2008;246:697-722.

10. Koo HJ, Lim S, Choe J, et al. Radiographic and CT features of viral pneumonia. Radiographics 2018;38:719-39.

11. Li Z, Zhang S, Zhang J, et al. editors. MVP-Net: Multiview FPN with Position-aware Attention for Deep Universal Lesion Detection. Shenzhen: International Conference on Medical Image Computing and ComputerAssisted Intervention, 2019.

12. Ronneberger O, Fischer P, Brox T, editors. U-net: Convolutional networks for biomedical image segmentation. Munich, Germany: International Conference on Medical image computing and computerassisted intervention, 2015.

13. Wang X, Zhang Q, Zhen Z, et al. Evaluating muticlass segmentation errors with anatomical priors. The IEEE International Symposium on Biomedical Imaging (ISBI) 2020. Available online: https://ieeexplore.ieee.org/ document/9098698

14. Bernheim A, Mei X, Huang M, et al. Chest CT Findings in Coronavirus Disease-19 (COVID-19): Relationship to Duration of Infection. Radiology 2020;295:200463.

15. Kong W, Agarwal PP. Chest imaging appearance of COVID-19 infection. Radiol Cardiothorac Imaging 
2020;2:e200028.

16. Shi H, Han X, Jiang $\mathrm{N}$, et al. Radiological findings from 81 patients with COVID-19 pneumonia in Wuhan, China: a descriptive study. Available online: https://www.thelancet. com/article/S1473-3099(20)30086-4/fulltext

17. Pan F, Ye T, Sun P, et al. Time Course of Lung Changes On Chest CT During Recovery From 2019 Novel Coronavirus (COVID-19) Pneumonia. Radiology 2020;295:715-21.

18. Tian S, Hu W, Niu L, et al. Pulmonary Pathology of Early-Phase 2019 Novel Coronavirus (COVID-19) Pneumonia in Two Patients With Lung Cancer. J Thorac
Oncol 2020;15:700-4.

19. $\mathrm{Xu} Z$, Shi L, Wang $\mathrm{Y}$, et al. Pathological findings of COVID-19 associated with acute respiratory distress syndrome. Lancet Respir Med 2020;8:420-2.

20. Wu J, Wu X, Zeng W, et al. Chest CT Findings in Patients With Coronavirus Disease 2019 and Its Relationship With Clinical Features. Invest Radiol 2020;55:257-61.

21. Yang Y, Lu Q, Liu M, et al. Epidemiological and clinical features of the 2019 novel coronavirus outbreak in China. medRxiv 2020:2020.02.10.20021675. Available online: https://www.medrxiv.org/content/10.1101/2020.02.10.200 $21675 \mathrm{v} 2$

Cite this article as: Hu X, Zeng W, Zhang Y, Zhen Z, Zheng Y, Cheng L, Wang X, Luo H, Zhang S, Wu Z, Sun Z, Li X, Cao Y, Xu M, Wang J, Chen W. CT imaging features of different clinical types of COVID-19 calculated by AI system: a Chinese multicenter study. J Thorac Dis 2020;12(10):5336-5346. doi: $10.21037 /$ jtd-20-1584 